\title{
Underlayer designs to enhance the performance of EUV resists
}

\author{
$\underline{\text { Hao Xu}}{ }^{\mathrm{a}}$, James M. Blackwell ${ }^{\mathrm{b}}$, Todd R. Younkin ${ }^{\mathrm{c}}, \mathrm{Ke} \mathrm{Min}^{\mathrm{b}}$ \\ ${ }^{a}$ Brewer Science, Inc., 2401 Brewer Drive, Rolla, MO 65401, USA \\ 'Intel's Molecules for Advanced Patterning (MAP) Program, LBNL Molecular Foundry, \\ 1 Cyclotron Road, Berkeley, CA 94720 USA \\ ${ }^{\mathrm{c}}$ Intel Corporation, $2501 \mathrm{NW} 229^{\text {th }}$ Avenue, Hillsboro, OR 97124 USA
}

\begin{abstract}
Extreme ultraviolet (EUV) lithography has gained momentum as the method of choice for $<32$-nm half-pitch device fabrication. In this paper, we describe our initial attempts to increase an EUV resist's sensitivity without compromising resolution and line roughness via introduction of a thermally crosslinkable underlayer. The main purpose is to test the possibility of using a combination of photoacid generators (PAGs) and EUV sensitizers (phenol type) in the underlayer designs to enhance the overall performance of EUV resists. We have demonstrated the possible benefits of adding an EUV underlayer into the regular EUV litho stack and investigated the effect of PAG types and loadings on the photospeed and litho performance of three different EUV resists.
\end{abstract}

Keywords: EUV, underlayer, lithography, photospeed, resolution, LWR, RLS tradeoff, photoacid generator, PAG

\section{INTRODUCTION}

EUV lithography (EUVL) is expected to offer a single-exposure solution for use in manufacturing at 22-nm half pitch, and possibly for some applications at 32-nm half pitch. ${ }^{1,2}$ However, in order to successfully implement EUVL in time, several technical hurdles must be overcome including: mask fabrication with low defects ${ }^{3}$; reliable EUV sources with high output power and sufficient lifetime ${ }^{4}$; contamination control of all mirrors ${ }^{5}$; high throughput; photoresists with sufficiently low line width roughness (LWR) and low exposure dose. For resist requirements, the international technology roadmap for semiconductors (ITRS) ${ }^{6}$ set targets for 3- $\sigma$ LWR at $<3 \mathrm{~nm}$ and for sensitivity at $<10 \mathrm{~mJ} / \mathrm{cm}^{2}$, which, to date, has not been achieved simultaneously in an EUV resist. Earlier research has actually found that most efforts simply result in a compromise between LWR, resolution, and sensitivity, e.g., good sensitivity but rough lines, or improved LWR but poor resolution. Although the design of new EUV photoresists and an improved understanding of the patterning mechanism has allowed significant progress for simultaneously achieving improved EUV resolution, LWR, and sensitivity (RLS), the trade-off is clearly a natural limitation for chemically amplified resists. ${ }^{7,8,9}$

In previous studies and publications, we have demonstrated the benefits of introducing an EUV underlayer beneath the resist. Specifically, we provided initial data on the relationship between film density/ adsorption and EUV litho performance. ${ }^{10}$ Additional benefits of using an EUV underlayer include, but are not limited to, planarization of a substrate with topography, protection of underlying layers from possible damage caused by high-energy EUV photons, improved adhesion for high-aspect-ratio resist profiles, and improved etch selectivity for better pattern transfer. Our goal in this paper is to use a spin-on EUV underlayer beneath the resist to harvest the extra energy from EUV photons. The energy is then redirected back to the resist in the form of photons, secondary electrons, or generated photoacids to enhance the resist's photosensitivity.

The underlayer is a crosslinkable polymeric system that can be coated onto a substrate through a regular spin-on process and then thermally cured so that the photoresist can be applied thereafter. Most underlayers have exhibited less outgassing than positive tone photoresists during EUV exposure. ${ }^{11}$ The polymer was chemically modified to have relatively high EUV adsorption and functional additives were included in the underlayer formulations to enhance the energy harvest and transfer process. In this paper, we will describe some of our efforts on various underlayer designs and their impacts on resist performance, mainly focusing on the possibility of using the combination of photoacid generators (PAGs) and EUV sensitizers (phenol type) in the underlayer to enhance EUV resist RLS performance.

Advances in Resist Materials and Processing Technology XXVI, edited by Clifford L. Henderson, Proc. of SPIE Vol. 7273, 72731J - @ 2009 SPIE · CCC code: 0277-786X/09/\$18 · doi: 10.1117/12.814223 


\section{EXPERIMENT}

\subsection{Material and Formulation}

All the underlayers studied in this paper are based on a crosslinkable linear polymer system. As shown in Figure 1 below, there are two basic polymer platforms involved in this study: a methacrylate platform A and polyhydroxy styrene (PHS) platform B. In platform A, various PAGs, including triphenylsufonium triflate (TPS-OTf), TPS nonaflate (TPSONf), and PAG-C1, were physically blended into the formulations to produce UL2, UL3, and UL4, respectively. In the case of UL4, different amounts of PAG-C1 were added to generate a series of UL4-10 (10 wt\%), UL4-16 (16 wt\%), and UL4-20 (20 wt\%). 2,5-dimethyl phenol (2,5-DiMe Ph) was added as an EUV sensitizer together with TPS-OTf to obtain UL5. ${ }^{12}$ Platform B bears triflate PAG and phenoxy groups on the polymer backbone, so no additional ingredients were needed to produce UL6. The polymer originally features $30 \%$ of PHS functionalized as a triflate, so the initial formulation using this polymer and crosslinker was named UL6-30. For comparison, PHS with no triflate moieties was blended in at one-half and two times the weight of this triflate functionalized PHS to make UL6-20 and UL6-10, which means $20 \%$ or $10 \%$ of the total PHS units were triflated, respectively.
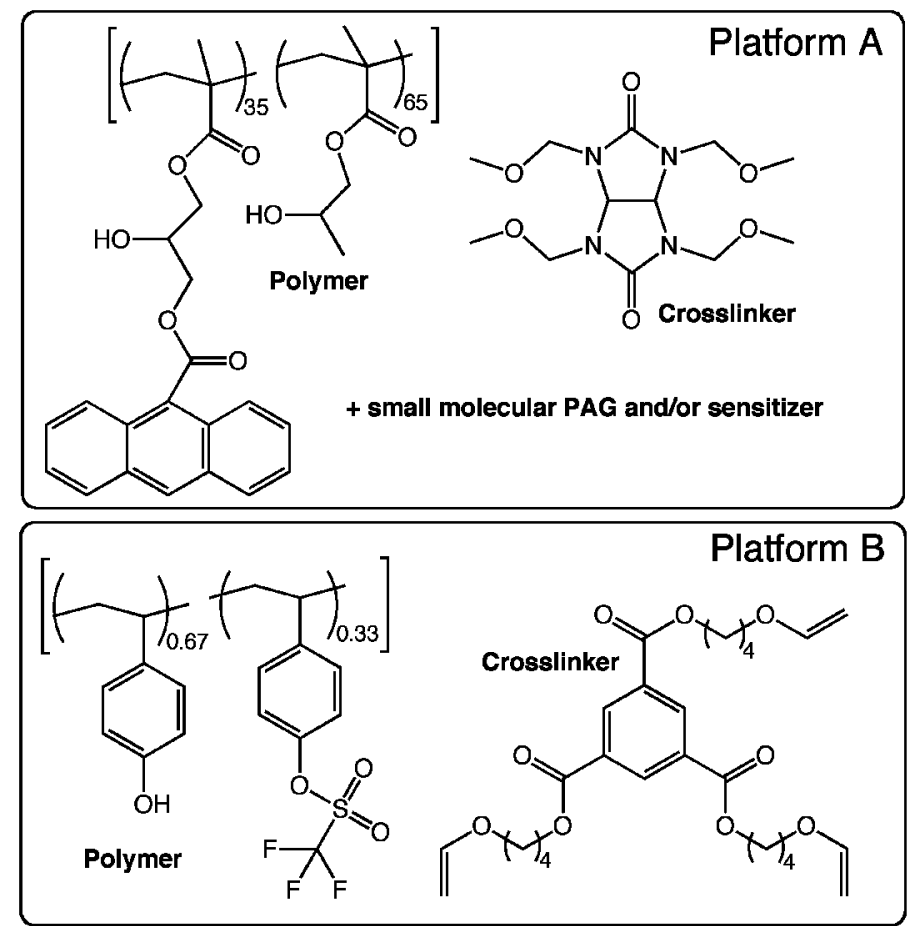

\begin{tabular}{|c|c|c|c|}
\hline Underlayers & Platform & PAG & EUV sensitizer \\
\hline UL1 & A & None & None \\
\hline UL2 & A & TPS-OTf & None \\
\hline UL3 & A & TPS-ONf & None \\
\hline UL4 (-10, -16,-20) & A & PAG-C1 & None \\
\hline UL5 & A & TPS-OTf & 2,5-DiMe Phenol \\
\hline UL6 (-10, -20, -30) & B & None & None \\
\hline
\end{tabular}

Figure 1. The chemical structures of two polymer platforms and six different underlayer formulations tested as part of this study.

\subsection{Process Condition and Measurement}

Polymer, crosslinker, and additives (if any) were dissolved completely in typical semiconductor-grade solvents (such as PGME, PGMEA, and combinations of the two) and filtered through a $0.1-\mu \mathrm{m}$ filter before use. Each of the final 
formulations (TSC $=1.5 \sim 2.5 \%$ ) was spin-coated at $1500-3000 \mathrm{rpm}$ onto a silicon substrate and then baked at $160^{\circ}$ $205^{\circ} \mathrm{C}$ for 60 seconds. The resultant film thicknesses ranged from 20 to $60 \mathrm{~nm}$, depending on the specific requirement of the lithography stack. The film was rinsed with ethyl lactate to test the film's solvent resistance (EL strip) and the film was also immersed in photoresist developer (TMAH base solutions) without light exposure to evaluate the dark loss. All the formulations used here were under 5\% EL stripping and dark loss without specification.

\subsection{Instrumentation}

\subsubsection{EUV Exposure and TOP CD SEM}

EUV exposures were performed on a microexposure tool (MET) either at the Advanced Light Source (ALS) in Berkeley or at Intel in Hillsboro, OR. The ALS-MET utilizes a 5x-reduction, $0.3-N A$ optic, providing a $200 \times 600 \mu \mathrm{m}$ exposure field at the wafer plane. All processing was performed using Berkeley BKMs as previously reported. ${ }^{13}$ The Intel-MET is a micro-field $600 \times 600 \mathrm{~mm}^{2}, 0.3$ numerical aperture (NA), two mirror lithography exposure tool, made by Exitech, Ltd. with resolution in excess of $30 \mathrm{~nm}$. The illumination settings used were annular with $\mathrm{s}$ inner $=0.36$ and $\mathrm{s}$ outer $=0.55$, normalized with respect to the pupil radius of 1 . All processing was done on $300 \mathrm{~mm}$ underlayer-coated Si wafers using a linked TEL-ACT12 track in Ronler Processing 1 (RP1), a Class 1 clean room fab. The CDs and LWR measurements were made using a CD Scanning Electron Microscope (SEM). The methodology used to measure LWR has been detailed previously. ${ }^{14}$ All of the LWR values reported in this article are an average of at least five to seven lines repeated at five different locations in the array. All photoresist testing was done using a $60 \mathrm{~nm}$ post-apply bake film thickness in order to maintain an aspect ratio of roughly 2:1 to help prevent pattern collapse. An aspect ratio of 2:1 balances the opposing needs to minimize resist thickness to reduce pattern collapse versus maximizing the resist thickness to help ensure that film absorbance is high enough such that sufficient aerial image contrast and photon absorption are achieved.

\subsubsection{Cross-section SEM}

The wafers were then sent to CeriumLabs in Austin, TX, for cross-section and imaging. The cross-section images shown in Figure 5 are after deposition with metals by the e-beam in the focused ion beam (FIB) and imaged using Hitachi S4800 high-resolution SEM/STEM.

\subsubsection{Time-of-flight Secondary-ion Mass Spectrometry (ToF-SIMS)}

ToF-SIMS is a surface-sensitive spectroscopy that uses a pulsed ion beam (Cs or microfocused $\mathrm{Ga}$ ) to remove molecules from the very outermost surface of the sample. The particles are removed from atomic monolayers on the surface (secondary ions). These particles are then accelerated into a "flight tube" and their mass is determined by measuring the exact time at which they reach the detector (i.e. time-of-flight). Depth profiles are produced by removal of surface layers by sputtering under the ion beam, which is used as the technique to probe the PAG distribution along the depth of the resist and underlayer.

\section{RESULTS AND DISCUSSION}

\subsection{The Effect of Adding Underlayers Beneath an EUV Resist}

It has been slowly recognized that a properly selected underlayer between substrate and EUV resist could contribute in enhancing the overall EUV resists' performance. UL1 is one of the first-generation underlayers, and we used it here as a baseline for studies described in this paper. As clearly shown in Figure 2, under the same tool setting, exposure condition, and photoresist, there is an obvious difference between the performance of TOK-P1123 coated directly on HMDS-primed silicon wafers and when coated onto UL1. The roughness of 40- and 32-nm half-pitch (HP) lines can be significantly decreased with an EUV underlayer present. When tested with Resist C at Intel, the LWR was also slightly reduced for both 40- and 30-nm HP lines by using UL1. However, at 32-nm HP the semi-isolated P1123 resist lines started to collapse on UL1, and the dose required for 30-nm HP Resist C patterning increased. Therefore, adding an EUV underlayer shows a trend of slightly reducing the line roughness, but may be subject to pattern collapsing and / or increased dose to size at 30-nm HP, depending on the EUV resists utilized. The explanation for LWR improvement by using an UL is not clear at this point, and is beyond the scope of this paper. The improvement of the adhesion to prevent 
pattern collapsing is under investigation now. Our main goal here is to understand, using UL1 as a control, if the addition of PAGs and/ or EUV sensitizer can increase the photosensitivity of the resist without negatively affecting LWR or resolution.

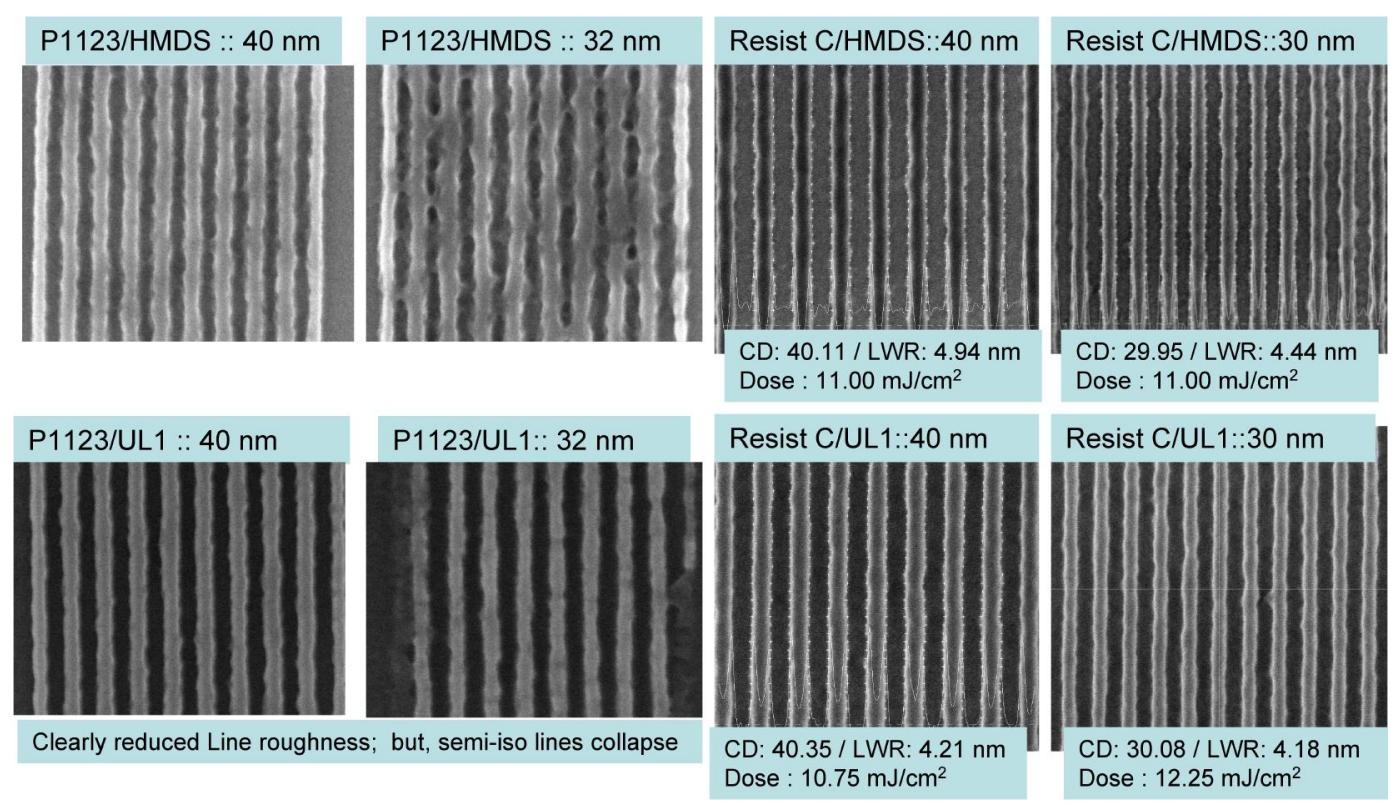

Figure 2. The comparison of top-down SEM images, at 40- / 32-nm, and 40- / 30-nm between HMDS-primed wafers and UL1, using either TOK-P1123 at ALS-MET Berkeley or Resist C at Intel-MET.

\subsection{The Photospeed Enhancement of EUV Resist by Adding PAGs and/ or EUV Sensitizers into the Underlayer}

In an effort to increase the photospeed of currently available EUV photoresist materials, we have investigated various additives to the baseline UL1. To increase resist photospeed, the key is to harvest as much energy as possible and then feed it back to the resist in different forms, e.g., photons, secondary electrons, or photo-generated acids. The first study was carried out by adding PAGs and energy sensitizers into the underlayer design in an effort to enhance the photoresist's performance, i.e., increased photospeed without compromising LWR and / or ultimate resolution.

Three different PAGs - TPS-OTf, TPS-ONf, and PAG-C1 - were added to UL1 at the same molar concentration. The same EUV resist (TOK-P1123) and illumination conditions were utilized in this study. Figure 3 shows the contrast curves for five different substrates, primed silicon substrate (HMDS), UL1, and UL1 doped with TPS-OTf (UL2), TPSONf (UL3), and PAG-C1 (UL4). Dose to clear $\left(\mathrm{E}_{0}\right)$ is defined as the dose at the point when film thickness (FT) decreases to $<1 \%$ of the original FT or when the curve slope starts to approach zero. The contrast (in the unit $=\mathrm{mJ}^{-1}$ ) was also calculated as the maximum slope of each contrast curve. Clearly, the addition of PAGs into the UL decreased the $\mathrm{E}_{0}$ significantly, by $16 \%$ in the case of the $\mathrm{C} 1 \mathrm{PAG}$. This is not too surprising because PAGs activated during exposure will generate photoacid that can help to clear the resist. An additional observation is that the C1 PAG has the largest effect while triflate shows the smallest, when added at the same molar concentration. The calculated slopes/ contrasts demonstrated the same trend with increased contrast from HMDS-primed wafer, to UL1, UL2, UL3, and UL4. We tentatively attribute this correlation to either acidic strength caused by various anions, or the different migration length/ depth into the top resist due to the inherent difference in molecular structure. However, more experiments are needed to reach a definite conclusion. 

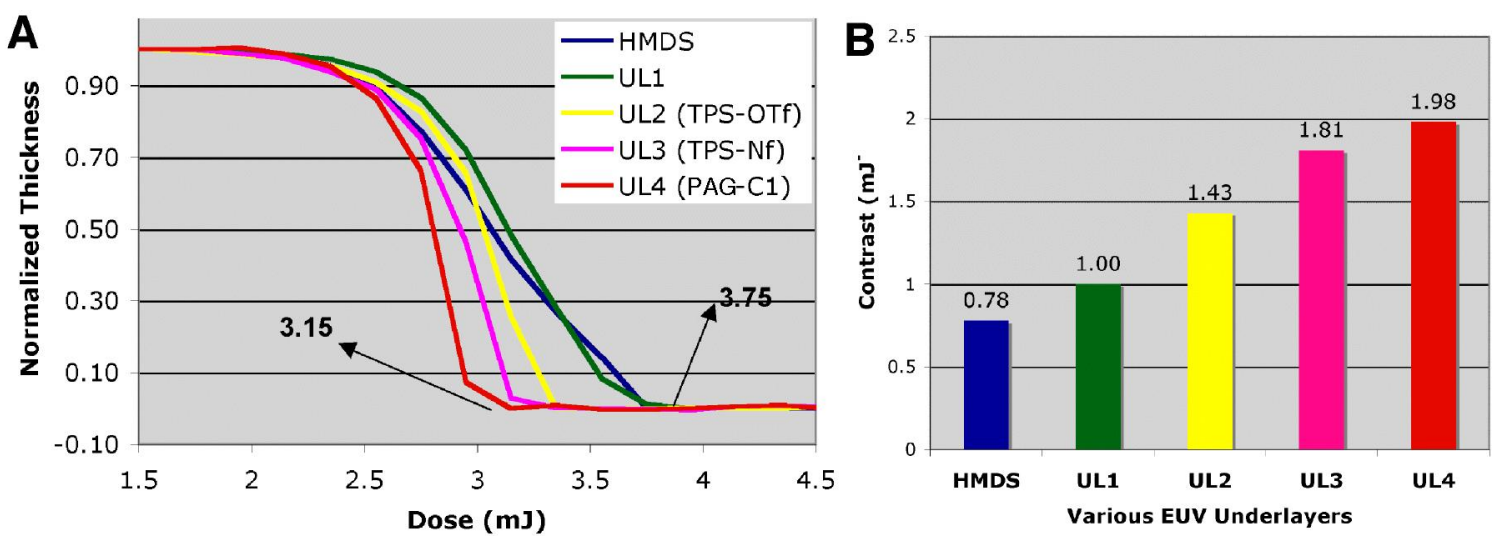

Figure 3. A) Contrast curves of TOK-P1123 on different substrates: HMDS-primed wafer, UL1, and underlayers with triflate (UL2), nonoflate (UL3), or C1 PAG (UL4) in the formulation. B) The slope of contrast curves.

Adding EUV sensitizers (phenol types) did not show effects as that were as obvious as the addition of PAGs. We explored different types of phenolic sensitizers, including but not limited to 2,5-dimethyl phenol, 4-nitro-phenol, 4methoxy-phenol, 2,6-dinitro-4-bromo-phenol, and various bispheno $1^{15}$ and polymer bounded phenols. Some of the phenols, such as 2,5-demehtyl phenol, have shown great effect in enhancing photospeed when added directly into photoresist. ${ }^{12}$ Unfortunately, only a slight differences in $\mathrm{E}_{0}$ with and without EUV sensitizers (phenols) in the underlayer design were observed, and no clear trends were present among different types of phenols at various concentrations. Part of the explanation might be due to the fact that most phenol molecules readily sublime, such that few are left in the matrix after our PAB. We also believe that the EUV sensitizers need to be in the proximity of the PAG to really see an effect, which is challenging for blended materials because of inhomogeneities and the high crosslinking density in UL1UL4. We will address this challenge in a later section of the paper.

\subsection{The General Effect of Added PAG and Sensitizer on Pattern Profiles}

To check the effect on line roughness and ultimate resolution, wafers were patterned using the ALS-MET at Berkeley with TOK-P1123. A 5 X 9 FEM was used, with $100-\mathrm{nm}$ focus step and $7 \%$ of central dose as dose step. Figure 4 shows the side-by-side comparison of 32- to 60-nm half-pitch top CD SEMs from UL1, UL2 (with TPS-OTf), and UL5 (with both TPS-OTf and 2,5-dimethyl phenol). There is some decrease in $\mathrm{E}_{\text {size }}$ with added PAG and/ or phenols, 7\%$14 \%$ varied at different pitches. However, the imaging quality deteriorated with the addition of PAG and/ or phenol. Especially at smaller pitches, the line roughness increased rapidly (7\%-26\%) and lines started to fall off at 32- and 34-nm HP. Clearly, the LWR and resolution were more compromised than the modest improvement in photospeed.

To more quantitatively analyze the data to examine the RLS trade-off, the concept of $Z$ factor was used to evaluate the balance of resolution, LER/ LWR, and photospeed. $\mathrm{Z}$ factor, as described elsewhere ${ }^{16}$, is defined as:

$$
\mathrm{Z} \text { factor }=(\text { resolution })^{3} *(\min \mathrm{LER})^{2} * \mathrm{E}_{\text {size }}
$$

The current target for 32-nm HP line/ space patterning is 2.0-nm 3- $\sigma$ LWR (or 1.4-nm LER) and $10 \mathrm{~mJ} / \mathrm{cm}^{2}$ dose to size, or Z-factor (32-nm target) $=6.6 \mathrm{E}-09$, which is defined as the target. A normalized value can then defined as $\mathrm{nZ}(32 \mathrm{~nm})$,

$$
\mathrm{nZ}_{32}=\mathrm{Z} \text { factor } / \mathrm{Z} \text { factor (32-nm target) }
$$

According to the Top SEM shown in Figure 4, the $\mathrm{nZ}_{32}$ calculated for each underlayer at the ultimate resolution are: 14.1 for UL1 at $34 \mathrm{~nm} \mathrm{HP}, 22.3$ for UL2 at $36 \mathrm{~nm} \mathrm{HP}$, and 17.4 for UL3 at $36 \mathrm{~nm}$ HP. Clearly there is no significant RLS improvement observed, although UL5 (PAG in combination with sensitizer) showed slightly better performance than UL2 (PAG only). (Table 1) 


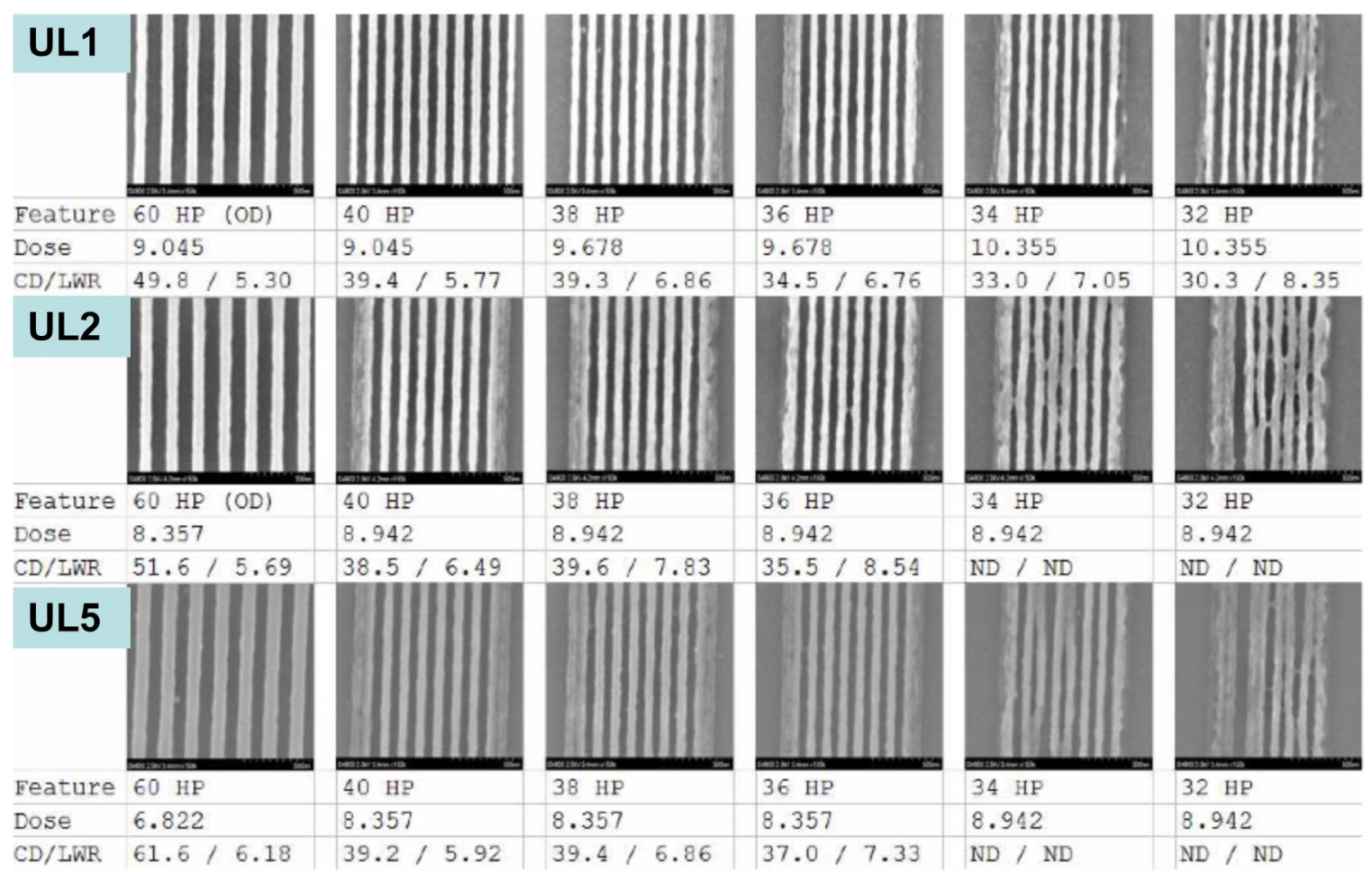

Figure 4. Top-down SEM images of EUVL on UL1, UL2, and UL5.

\begin{tabular}{|c|c|c|c|}
\hline & Ultimate resolution & Z-Factor $\left(\mathbf{m J} \mathbf{J}^{\mathbf{*}} \mathbf{)}\right.$ & $\mathbf{n Z}_{\mathbf{3 2}}$ \\
\hline UL1 & $34 \mathrm{~nm}$ & $9.30 \mathrm{E}-08$ & 14.1 \\
\hline UL2 & $36 \mathrm{~nm}$ & $1.47 \mathrm{E}-07$ & 22.3 \\
\hline UL5 & $36 \mathrm{~nm}$ & $1.15 \mathrm{E}-07$ & 17.4 \\
\hline
\end{tabular}

Table 1. The normalized $\mathrm{Z}$ factors on three different ULs.

Cross-section SEM images were taken at the best dose and focus for 36-, 40-, and 60-nm HP to evaluate the pattern profile and line shape. As shown in Figure 5, footing was observed with UL1, and became more severe as CD size decreased from $60 \mathrm{~nm}$ to $40 \mathrm{~nm}$ to $36 \mathrm{~nm}$. Adding PAG and/ or phenols not only decreases the required dose to size, which is in agreement with top CD SEM observations, but also helps to keep the resist's profile straight by reducing footing and scumming. To investigate the cause of footing and how a PAG in the UL formulation helps, TOF-SIMS was performed to track fluorine (F)/ PAG distribution along film depth to the substrate, as all three PAGs used here bear the distinctive $\mathrm{F}$ atom compared to the rest of the hydrocarbon-based resists/ ULs. The SIMS data first confirmed the composition of these four different ULs: no PAG in UL1, and different PAGs in UL2, UL3, and UL4 with equal molar concentrations. Because triflate has only $3 \mathrm{~F}$ atoms per molecule while both nonaflate and $\mathrm{C} 1 \mathrm{PAG}$ have $9 \mathrm{~F}$ atoms per molecule, it is not surprising that the F peak in UL2 is much lower than in UL3 and UL4. It is also clear that the distribution of PAG inside the resist is not uniform, with a deficient area near the photoresist / UL interface, which at least partially explains the formation of footing. Therefore, the photoacids generated and then diffused from the PAGs of the top UL can balance this deficiency, and keep the resist profile straighter. However, untamed PAG/ photoacid diffusion from the UL can also causes line collapse, especially semi-isolated lines, which explains why the ultimate resolution was not as good as the baseline UL1, where no PAG was added. 

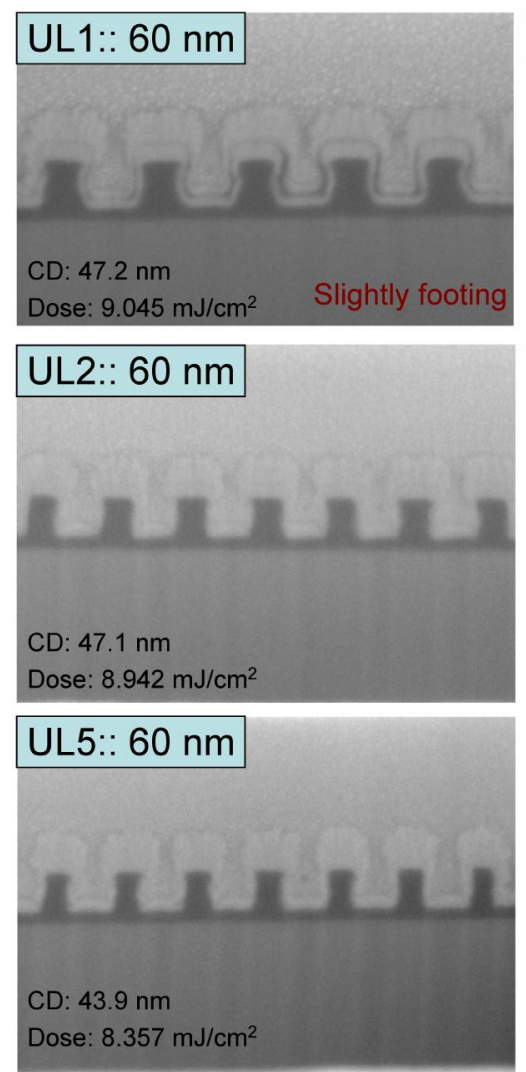
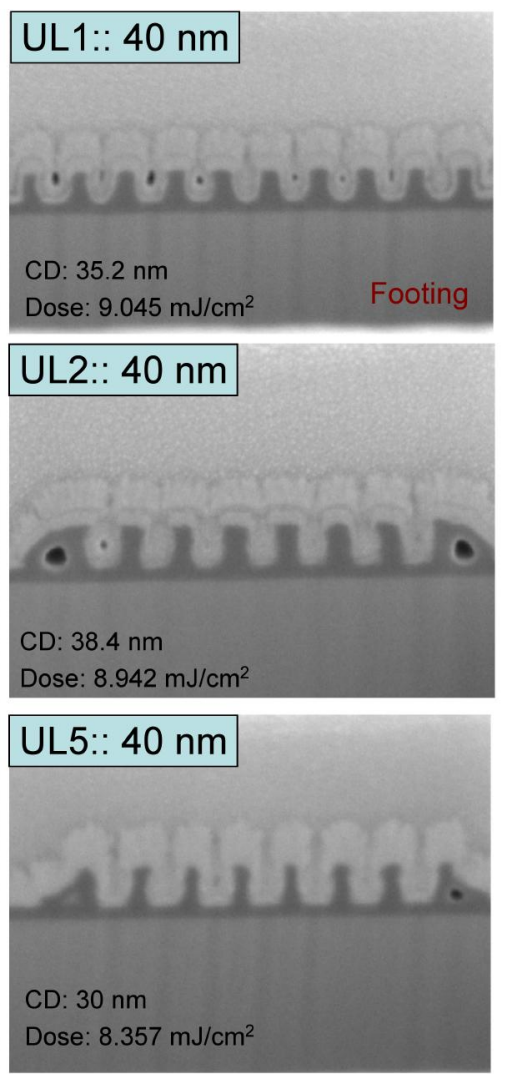
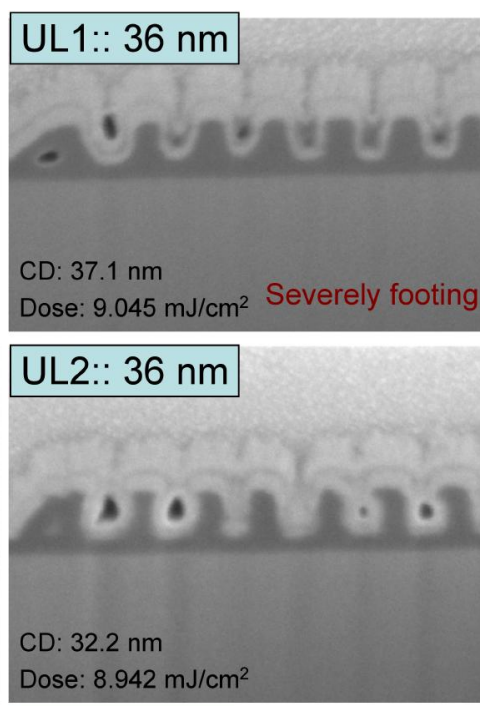

UL5:: $36 \mathrm{~nm}$

CD: NA

Dose: $8.357 \mathrm{~mJ} / \mathrm{cm}^{2}$ Line collapsing

Figure 5. Cross-section SEM images of 60-, 40-, and 36-nm HP lines on UL1, UL2, and UL5.

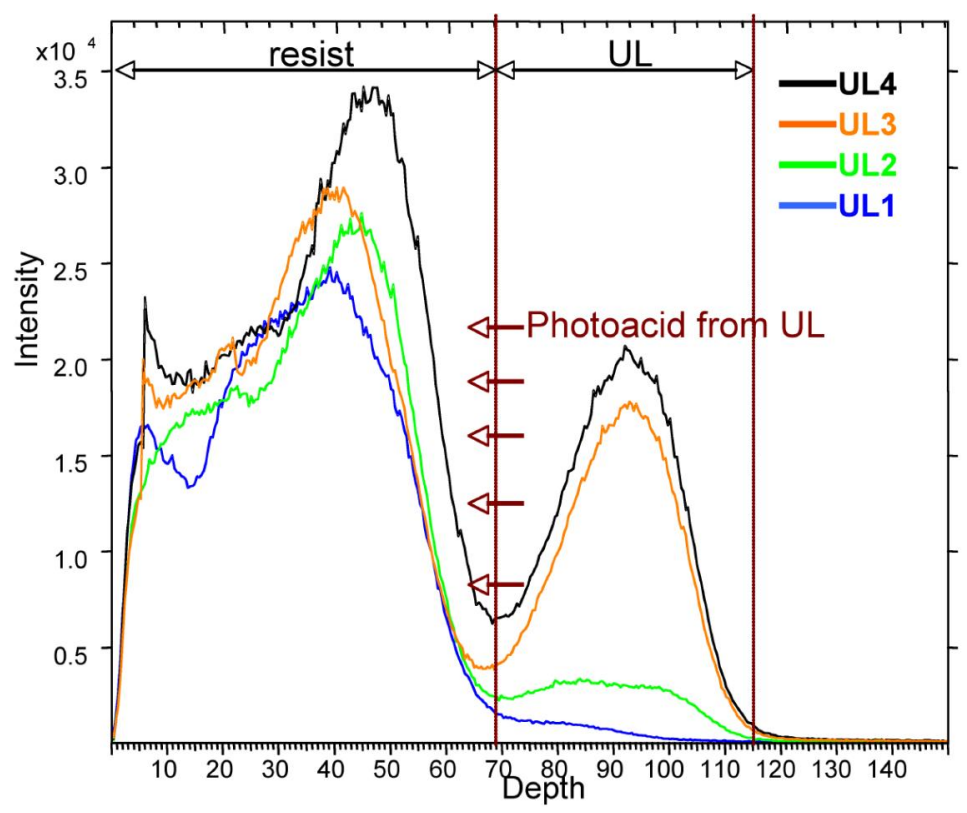

Figure 6. SIMS profile of TOK-P1123 (70 nm) resist on UL1, UL2, UL3, and UL4 (40 nm). 


\subsection{The Effect of PAG Amount on Dose to Size of Resist}

To examine the effect of different PAG loadings, various amounts of PAG-C1 were used in the formulation of UL4 at the levels of $10-\mathrm{wt} \%, 16-\mathrm{wt} \%$, and $20-\mathrm{wt} \%$. The resulting formulations were called UL4-10, UL4-16, and UL4-20, respectively. In this study, all underlayers were coated at $60 \mathrm{~nm}$ FT using Resist C. Dose to clear $\left(\mathrm{E}_{0}\right)$ and dose to size at 30-nm HP $\left(E_{30}\right)$ were evaluated with Intel's MET tool. First of all, a $24 \% \mathrm{E}_{0}$ decrease was observed, which is in agreement with previous results at ALS LBL using TOK-P1123 resist. Clearly the increased photospeed resulting from the addition of PAG to the UL was independent of the exposure tool and EUV resist used. Second, a $4 \% \mathrm{E}_{30}$ decrease further confirmed that the photospeed increase is not only a macroscopic phenomenon but also happens at the microscopic scale. Another important trend is that increasing PAG loading does not generate a greater photospeed increase; rather both $\mathrm{E}_{0}$ and $\mathrm{E}_{30}$ saturated at a certain value after $10-\mathrm{wt} \%$ PAG additions. From the PAG distribution indicated in Figure 6, the possible explanation might be that increased PAG loading will only increase the peak height (central concentration) inside the UL and not increase the number of photoacids that can actually diffuse into the top resist. The real PAGs that contribute lie only at the interface, and their concentrations are not significantly effected by the total PAG concentration inside the UL. This is a very valuable observation for future investigative direction: focusing on the increase of local PAG concentration at the resist/ UL interface.

\begin{tabular}{|c|c|c|c|c|}
\hline & UL1 & UL4-10 & UL4-16 & UL4-20 \\
\hline Dose to clear $\left(\mathrm{E}_{\mathbf{0}}\right)$ & 6.25 & 4.75 & 4.75 & 4.75 \\
\hline Dose to 30nm ( $\left.\mathbf{E}_{\mathbf{3 0}}\right)$ & 12.25 & 11.75 & 11.75 & 11.75 \\
\hline
\end{tabular}

Table 2. The dose to clear and dose to size 30-nm HP (in $\mathrm{mJ} / \mathrm{cm}^{2}$ ) of Resist C on UL1, UL4-10, UL4-16, and UL4-20.

\subsection{PAG Comparison Between Triflate and PAG C1}

In an effort to utilize the PAG in a more controlled way to maintain the benefit of decreased $\mathrm{E}_{\text {size }}$ without sacrificing LWR and resolution, a PAG utilizing a bulkier anion was employed. TPS-triflate has been known to have a long diffusion length that can lead to unwanted line collapse and lifting. PAG C1 with a branched multifluorocarbon functionalized anion, was used in the following study in comparison with TPS-triflate to see if the selection of PAG would lead to a difference in patterning quality. Wafers were patterned using the Intel-MET with Resist C. An 11 X 21 FEM was used, with $40-\mathrm{nm}$ focus step and $0.75-\mathrm{mJ} / \mathrm{cm}^{2}$ dose step.

The results are shown in Figure 7, comparing the $\mathrm{E}_{30}$, LWR, Z-factor, and depth of focus (DOF) for UL1, UL2 (triflate), and UL4 (PAG C1). Because of the different resist and tool used this time, the comparison between UL1 and UL2 is slightly different from what was observed in Figure 4. However, there is still no convincing increase in $\mathrm{Z}$ factor from the addition of TPS-triflate to the UL. Focusing on the difference between triflate and PAG C1, it is easy to notice that UL4 with PAG C1 did generate an obvious advantage over UL2 with triflate PAG - smoother lines, smaller Z-factor, and bigger DOF for the same $\mathrm{E}_{\text {size. }}$. This actually confirmed our initial assumption that by using an equal amount of PAG C1 instead of triflate, the same photospeed increase could be achieved while not greatly impacting LWR and resolution due to a more controllable diffusion length of a bulky anion. 


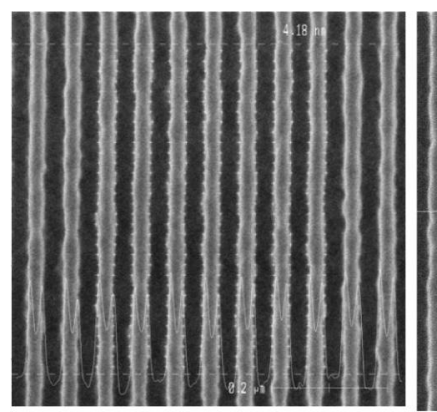

UL1 (no PAG)

$E_{30}=12.25 \mathrm{~mJ} / \mathrm{cm}^{2}$

Min LWR $=4.2 \mathrm{~nm}$

$Z$-factor $=2.9 \mathrm{E}-08$

$n Z(32)=4.4$

DOF $(30 \mathrm{~nm})=0.27$

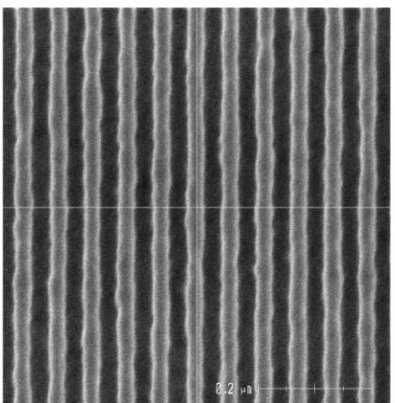

UL2 (w / Triflate)

$\mathrm{E}_{30}=11.75 \mathrm{~mJ} / \mathrm{cm}^{2}$

Min LWR $=4.2 \mathrm{~nm}$

Z-factor $=2.8 \mathrm{E}-08$

$n Z(32)=4.2$

DOF $(30 \mathrm{~nm})=0.25$

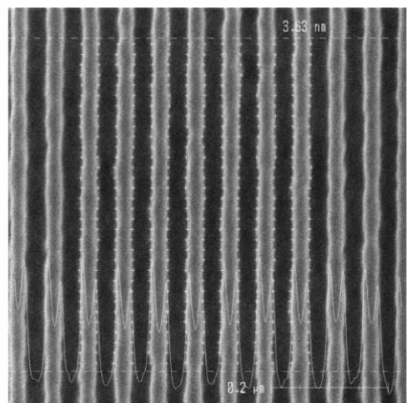

UL4-16 (w/C1)

$E_{30}=11.75 \mathrm{~mJ} / \mathrm{cm}^{2}$

Min LWR $=3.6 \mathrm{~nm}$

$Z$-factor $=2.1 \mathrm{E}-08$

$n Z(32)=3.2$

DOF $(30 \mathrm{~nm})=0.3$

Figure 7. The comparison of Resist C performance on UL1, UL2, and UL4-16.

\subsection{An Improved System - Platform B and UL6 Series}

Through the initial studies on the Platform A-based system, we found that, due to uncontrolled photoacid diffusion, the benefit we gained in photospeed increase was compromised by the loss of resolution and an increase in line edge roughness. In addition, it appears that in order to make the EUV sensitizer work together with PAG, they needs to be put in close proximity to each other. Finally, physically blending small molecules in the underlayer formulation does not seem very efficient and is hard to control. Based on these three observations, we designed a new polymer system that includes a crosslinkable polymer matrix, a phenolic-type sensitizer anchored to the polymer backbone, and a "cationbound" covalently attached PAG, shown as Platform B in Figure 1. With such a design, first, the PAG is bound to the polymer so that it is more evenly distributed in the underlayer and less likely to leach into the photoresist during resist coating; second, the phenol is part of the polymer such that it is not as apt to sublime as most phenol-type small molecules; and third, the PAG and phenol are held in proximity to each other for more efficient energy transfer.

In this study, an open-source resist - TER60AB - was used, which comprises a $60 \%$ hydroxystyrene, 20\% styrene, $20 \%$ t-butyl acrylate terpolymer, TPS-triflate as PAG, and trioctylamine as a base quencher. Although it is not the bestperforming EUV resist, its open-source structure and composition gave us the opportunity to understand how the resist and underlayer interact together.

During the initial tests, it was found that UL6-10 gave the best performance, as shown in Figure 8. Due to a dose shift during the exposure slot, there is no exact dose information associated with each SEM, but through a reasonable estimation, we believe the maximum dose would be $\sim 9 \mathrm{~mJ} / \mathrm{cm}^{2}$. If this assumption is correct, the SEM of 32-nm HP shown below is much better than the 32-nm lines illustrated in Figure 4, i.e., the lines are smoother with reduced pattern collapse. The $\mathrm{Z}$ factor calculated is $6.8 \mathrm{E}-08$ and $\mathrm{nZ}_{32}=10.3$, much closer to the $32-\mathrm{nm} \mathrm{HP}$ target. In addition, the ultimate resolution extended to $28-\mathrm{nm} \mathrm{HP}$ (with $26.8-\mathrm{nm}$ actual $\mathrm{CD}$ ). By calculation, the $\mathrm{Z}$ factor is $5.8 \mathrm{E}-08$ and $\mathrm{nZ}_{32}=$ 8.9. With an open-source triflate-based resist, this result is very encouraging, and we are in the process of improving the structure design and formulation. 


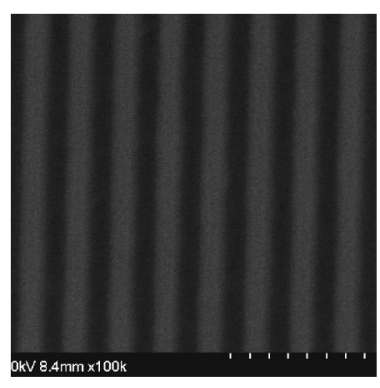

$\mathrm{CD}=45.8 \mathrm{~nm}$

$\mathrm{LWR}=8.9 \mathrm{~nm}$

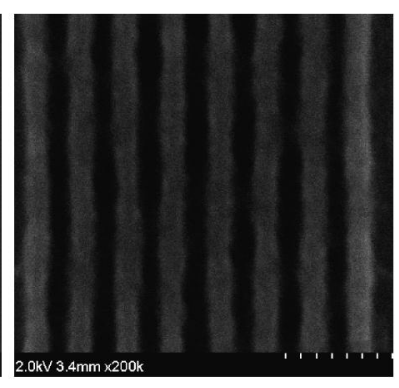

$\mathrm{CD}=31.3 \mathrm{~nm}$

$\mathrm{LWR}=7.0 \mathrm{~nm}$

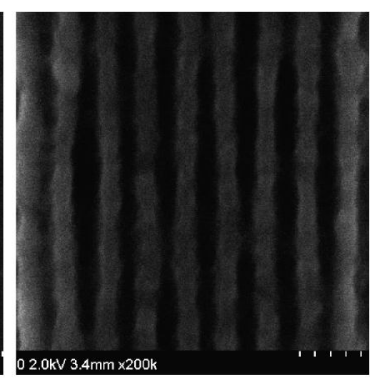

$\mathrm{CD}=26.8 \mathrm{~nm}$

$\mathrm{LWR}=8.2 \mathrm{~nm}$

Figure 8. SEM images of open-source resist TER60AB on UL6-10 at 40-, 32-, and 28-nm HP lines.

\section{SUMMARY}

In summary, to test the idea of improving EUV resists' performance by introducing an EUV underlayer with specially designed additives, six types (10 actual formulations) of underlayers were investigated with three different resists (TOKP1123, Resist C, and open-source TER60AB) on MET EUV exposure tools at both Berkeley and Intel. The use of an appropriate underlayer can clearly impose some benefits on the overall EUVL performance (e.g. slightly decreased LWR), independent of the resist and tool used. The addition of PAG into an EUV underlayer reproducibly showed the ability to increase the photospeed of the resists, i.e., $7 \%-25 \%$ decrease of $E_{0}$ and $E_{\text {size. }}$. Underlayers with PAG (or together with sensitizer) can also reduce footing and scumming between patterned lines, keeping the resist shape more square and straight. However, untamed photoacid diffusion still causes this improvement to be subject to the RLS tradeoff by compromising LER and resolution. Therefore, we have not observed a significant RLS gain using the underlayers reported herein. Recent designs that incorporate PAG and possible EUV sensitizers into the polymer backbone provided very promising initial results. Further investigation is underway and will be reported in the future.

\section{ACKNOWLEDGEMENT}

The authors would like to thank Huiying Liu (Intel) for TOF-SIMS analysis and JSR Corporation / JSR Micro, Inc. for donation of the TER60 resist employed in this study.

\section{REFERENCES}

1. Wurm, S., "Outlook: EUVL lithography manufacturing insertion,” Solid State Technology, October (2006).

2. Cobb, J. L., Brainard, R. L., O'Connell, D. J., and Dentinger, P. M., "EUV Lithography: Patterning to the end of the road," Mat. Res. Soc. Symp. Proc. 705, Y3.2.1-Y3.2.10 (2002).

3. Gullikson, E. M., Cerjan, C., Stearns, D. G., Mirkarimi, P. B., and Sweeney, D. W., "Practical approach for modeling extreme ultraviolet lithography mask defects," J. Vac. Sci. Technol. B, 20, 81-86 (2002).

4. Bakshi, V., "EUV sources for lithography," SPIE press, Bellingham, WA (2005).

5. Mirkarimi, P. B., Spiller, E., Baker, S. L., Sperry, V., Stearns, D. G., and Gullikson, E. M., "Developing a viable multilayer coating process for extreme ultraviolet lithography reticles," J. Microlith., Microfab., Microsyst. 3, 139-145. (2004).

6. International Technology Roadmap for Semiconductors, 2007 Edition, Lithography.

7. Brainard, R. L., Trefonas, P., Lammers, J. H., Cutler, C. A., Mackevich, J. F., Trefonas, A., Robertson, S. A., "Shot noise, LER, and quantum efficiency of EUV photoresists," Proc. SPIE, 5374 (Pt. 1), 74-85 (2004).

8. Gallaitin, G. M., Naulleau, P., Brainard, R., "Fundamental limits to EUV photoreist," Proc. SPIE, 6519, 651911/1651911/10 (2007).

9. Schmid, G. M., Stewart, M. D., Wang, C., Vogt, B. D., Prabhu, V. M., Lin, E. K., Willson, C. G., "Resolution limitations in chemically amplified photoresist systems," Proc. SPIE, 5376, 333-342 (2004). 
10. Guerrero, D. G., Xu, H., Beaman, C., Sakamoto, R., Endo, T., Ho, B.-C., "Effects of underlayers on EUV lithography" [poster], 2008 International EUVL Symposium [SEMATECH], Lake Tahoe, CA, Sept. 28-Oct. 1, 2008.

11. Douglas J. Guerrero, Carol Beaman, Rikimaru Sakamoto, Takafumi Endo, and Bang-Ching Ho, "Organic underlayers for EUV lithography," Journal of Photopolymer Science and Technology, vol. 21, no. 3, 541-455 (2008).

12. Kozawa, T., Tagawa, S., "Acid distribution in chemically amplified extreme ultraviolet resist," J. Vac. Sci. Technol. $B, 25,2481,(2007)$.

13. Ma, A., Park, J., Dean, K., Wurm, S., Naulleau, P., "Benchmarking Commercial EUVL Resists at SEMATECH," Proc. of SPIE, 6921, 692130 (2008)

14. Chandhok, M., Frasure, K., Putna, E.S., Younkin, T., Rachmady, W., Shah, U., and Yueh, W., "Improvement in Linewidth Roughness by Postprocessing," J. Vac. Sci. Technol. B 262265 (2008).

15. Hermann, R., Naumov, S., Mahalaxmi, G. R., Brede, O., "Stability of phenol and tiophenol radical cations interpretation by comparative quantum chemical approaches," Chem. Phy. Lett. 324, 265-272 (2000).

16. Wallow, T., Higgins, C., Brainard, R., Petrillo, K., Montgomery, W., Koay, C., Denbeaux, G., Wood, O., Wei, Y., "Evaluation of EUV resist materials for use at the 32 nm half-pitch node," Proc. of SPIE, Vol. 6921, 69211F, (2008) 